TecnoLógicas

ISSN-p 0123-7799

ISSN-e 2256-5337

Vol. 23, No. 47, pp. 121-136

Enero-abril de 2020

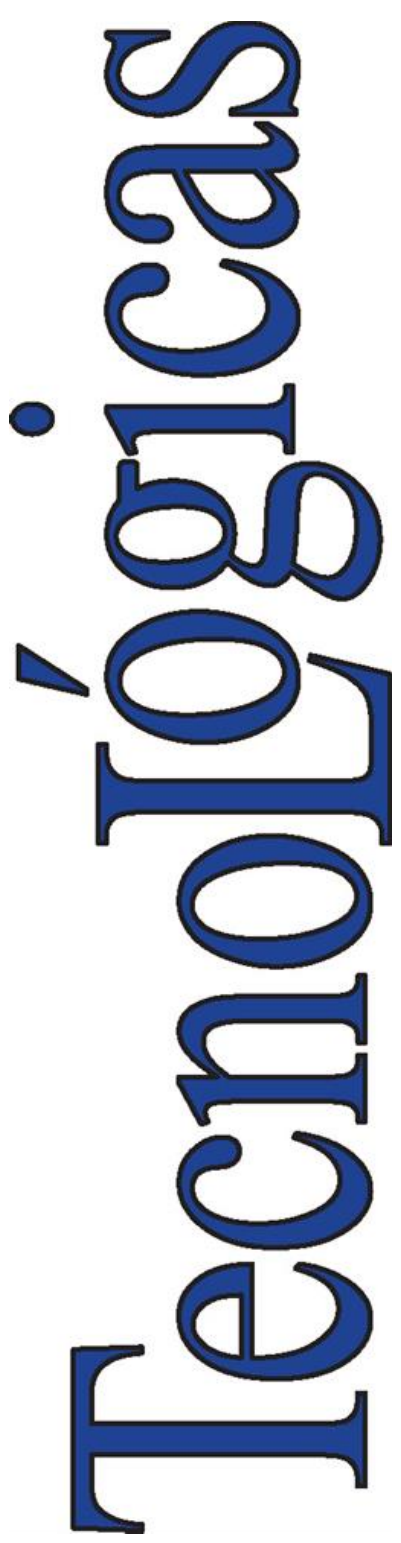

(C) Instituto Tecnológico Metropolitano Este trabajo está licenciado bajo una Licencia Internacional Creative Commons Atribución (CC BY-NC-SA)

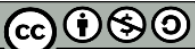

Artículo de Investigación/Research Article

\section{Design of a Wireless Sensor Network for Optimal Deployment of Sensor Nodes in a Cocoa Crop}

\section{Diseño de una red de sensores inalámbricos para el despliegue óptimo de los nodos sensores en un cultivo de Cacao}

\author{
Jose M. Celis-Peñaranda (D) 1 , \\ Christian D. Escobar-Amado(iD2, \\ Sergio B. Sepúlveda-Mora (D) ${ }^{3}$, \\ Sergio A. Castro-Casadiego(D), \\ Byron Medina-Delgado(iD 5 y \\ Daniel Guevara-Ibarra (D)6
}

Recibido: 29 de mayo de 2019 Aceptado: 16 de octubre de 2019
Cómo citar / How to cite:

J. M. Celis-Peñaranda, C. D. Escobar-Amado, S. B. Sepúlveda-Mora, S. A. Castro-Casadiego, B. Medina-Delgado, D. Guevara-Ibarra, "Design of a Wireless Sensor Network for Optimal Deployment of Sensor Nodes in a Cocoa Crop”. TecnoLógicas, vol. 23, no. 47, pp. 121-136, 2020. https://doi.org//10.22430/22565337.1361

1

Electronics Engineer, Electricity and Electronics department, GIDET Universidad Francisco de Paula Santander, Cúcuta-Colombia, josemiguelcp@ufps.edu.co

2 Electronics Engineer, Electricity and Electronics department, GIDET, Universidad Francisco de Paula Santander, Cúcuta-Colombia, christiandavidea@ufps.edu.co

3 MSc. of Science in Electrical and Computer Engineering, Electricity and Electronics department, GIDET, Universidad Francisco de Paula Santander, Cúcuta-Colombia, sergio.sepulveda@ufps.edu.co

4 MSc. in Electronics Engineering, Electricity and Electronics department, GIDET, Universidad Francisco de Paula Santander, Cúcuta-Colombia, sergio.castroc@ufps.edu.co

5 MSc. in Electronics Engineering, Electricity and Electronics department, GIDET, Universidad Francisco de Paula Santander, Cúcuta-Colombia, byronmedina@ufps.edu.co

6 PhD. in Engineering, Electricity and Electronics department, GIDET, Universidad Francisco de Paula Santander, Cúcuta-Colombia, dinaelgi@ufps.edu.co 


\begin{abstract}
In this study, factorial experiments were conducted in two different scenarios to design a Wireless Sensor Network for monitoring a cocoa crop in a rural area in Colombia. Node sensors measured temperature, relative humidity, soil moisture, Ultra-Violet light, and visible light intensity. The factors considered in the experiments were distance between node sensors, height from the ground, and type of antenna; in turn, Received Signal Strength Indicator and data transfer time were the outputs. The wireless sensor network was deployed in the crop, covering approximately $3 \%$ of the area and using 7 different nodes in a cluster tree topology. First, an open field scenario with line of sight was used to determine the appropriate height of the node sensors. Second, a scenario in the actual cocoa crop was utilized to find the appropriate distance between modules and type of antenna. We found, based on our calculations and experimental data, that a height of $1.25 \mathrm{~m}$ was required to avoid the Fresnel zone and improve the RSSI of the network. Furthermore, we determined that a distance below $35 \mathrm{~m}$ was needed to guarantee signal reception and avoid long data transfer times. The wire antenna exhibited a better performance. Finally, the proposed methodology and monitoring system can be used for agronomic applications in rural areas in Colombia to increase crop yield.
\end{abstract}

\title{
Keywords
}

Wireless sensor networks, factorial experiments, agronomic crop, XBee module, ZigBee wireless.

\section{Resumen}

En este trabajo se realizaron experimentos factoriales en dos escenarios diferentes, para diseñar una red de sensores inalámbricos, que permita monitorear un cultivo de cacao en una zona rural de Colombia. Los nodos sensores miden la temperatura, la humedad relativa, la humedad del suelo, la luz ultravioleta y la intensidad de la luz visible. Los factores considerados en los experimentos fueron la distancia entre los nodos sensores, la altura con respecto al suelo y el tipo de antena; el indicador de intensidad de señal recibida y el tiempo de transferencia de datos fueron las salidas. La red de sensores inalámbricos se implementó en el cultivo, cubriendo aproximadamente el $3 \%$ del área, utilizando 7 nodos diferentes en una topología de cluster-tree. En primer lugar, se utilizó un escenario de campo abierto con línea de vista para determinar la altura adecuada de los sensores de nodo. Luego, se utilizó un escenario en el cultivo de cacao real para encontrar la distancia adecuada entre los módulos y el tipo de antena. Se obtuvo, por cálculos y datos experimentales, que se requería una altura de $1.25 \mathrm{~m}$ para evitar la zona de Fresnel y mejorar el RSSI de la red. Además, se determinó que se necesitaba una distancia inferior a $35 \mathrm{~m}$ para garantizar la recepción de la señal y evitar largos tiempos de transferencia de datos. Adicionalmente, la antena tipo Wire exhibió un mayor rendimiento y la metodología propuesta y el sistema de monitoreo se pueden usar para aplicaciones agronómicas en áreas rurales de Colombia, con el fin de aumentar el rendimiento de los cultivos.

\section{Palabras clave}

Red de sensores inalámbricos, experimentos factoriales, cultivo agronómico, módulo XBee, ZigBee inalámbrico. 


\section{INTRODUCTION}

PLANT Physiology is the study of the life processes of plants, how and why each plant behaves in its own particular way. It is the study of the organization and operation of the processes that define their development and behavior. Each plant is the product of its genetic information modified by its environment, and each plant organ is further modified by the physiological state or internal environment of the plant. Physical and chemical laws explain how plants are able to use inorganic substances and the energy of light to synthesize organic molecules that form their complex structures [1].

Appropriate plant growth depends on specific conditions of the soil and climate variables; therefore, monitoring environmental conditions is crucial for maximizing crop yield and minimizing diseases that affect crops. Common variables of interest in a crop are temperature, humidity, and light [2]. Agricultural production systems based on the Internet of things (IoT) and using correlation analysis between statistical information of the crop and information from the environment have improved the ability of farmers, researchers, and government officials to analyze current conditions and predict the upcoming yield of crops [3].

Monsalve, Arias, and Mejia concluded that wireless networks can serve as communication systems in control applications with allowable delays of up to 50 ms. In addition, their results show a better performance of Industrial Ethernet networks over conventional networks, with differences in the RTT (Round-Trip Time) of milliseconds [4]. Monitoring environmental variables in greenhouses or crops is decisive to identify issues in the crop yield. Therefore, different parameters must be measured to be processed and stored; this data collection allows farmers and researchers to study the behavior of the plants in the crop.
This monitoring task can be performed by a Wireless Sensor Network (WSN), which consists of autonomous, spatially distributed devices that use sensors to monitor physical or environmental conditions. A WSN system incorporates a gateway that provides wireless connectivity to distributed nodes. The wireless protocol of the WSN depends on the requirements of the application. Some of the available standards include $2.4 \mathrm{GHz}$ radios based on IEEE 802.15.4 or IEEE 802.11 (WiFi) standards or proprietary radios, which regularly operate at $900 \mathrm{MHz}$. A wireless communications protocol used in WSNs is ZigBee, established by the ZigBee Alliance and compatible with more than 70 associated companies [5]. It was developed to meet the needs of safety, reliability, flexibility, low cost, and low power consumption of different areas of process control, which is why this protocol is considered the most promising for wireless sensors.

By using WSNs, IoT can be integrated into agriculture, which leads to the interaction of agronomists, farmers, and crops regardless of their geographical separation [6], offering remote supervision from anywhere in the world.

Control systems in traditional greenhouses are mainly based on cable communication, which has some drawbacks, such as the complexity of wiring, maintenance, inflexibility of updates, and deployment, among others [7]. Many authors have used WSNs as monitoring systems; for instance, Cama-Pinto et al. utilized a WSN to measure humidity, temperature, light, and volumetric water content in the soil. The WSN sent the data collected from the sensors to an embedded device where the information was stored in a data base, so that real time values of the variables in the crop could be visualized in a graphic user interface [8].

The use of wireless sensors overcomes the aforementioned drawbacks and does not interfere with harvesting activities, which may reduce implementation costs. WSNs can be implemented with open source embedded systems; for example, Nikhade employed the 
Raspberry Pi card as a base node in a sensor network, improving the versatility of the system's architecture. This embedded system was used to process, analyze, monitor, and create a supervisory interface for the data [9].

Several studies have been conducted worldwide to monitor and control different agronomic crops, study their behavior, and control environmental conditions. Azimi et al. [10] developed and implemented a control system for environmental variables in mushroom crops. The system had environmental sensors DHT11 and MQ135 to measure temperature, humidity, and carbon dioxide. The system they proposed also included six Wi-Fi ESP8266 modules, connected with ThingSpeak.com to send the measured data, and a separate module that received the results of the analysis and controlled the irrigation system [10]. Similarly, Subashini et al. [11] developed an irrigation control system based on an 8-bit AVR microcontroller using low-cost sensors and actuators. The variables they used in their study were humidity, ambient and soil temperature, and light intensity. The processing system was connected to an ESP8266 Wi-Fi module that communicated with an Internet server, to which the results of the measurements were sent so that they could be stored and analyzed together with climate data. This optimized the irrigation cycles of the crop [11]. In turn, Sung et al. [12] analyzed, monitored, and controlled environmental variables that influenced the development of a plant. They implemented a Wireless Sensor Network (WSN) with XBee modules, Seeduino Stalker cards, and sensors to measure temperature, environment and soil humidity, air quality, and light. In the tests, plants were exposed to two different types of radiation: sunlight and artificial illumination. The physical development of the plant was compared with that of another plant of the same species to which the supervised control of environmental variables had not been applied [12].
Pule et al. [13] surveyed the applications of WSN in environmental monitoring with a focus on water quality. They also compared and evaluated sensor node architectures proposed by various authors in terms of monitored parameters, microcontroller/ microprocessor units (MCU) and wireless communication standards, localization, data security implementation, power supply, architectures, autonomy, and potential application scenarios [13]. It is also important to achieve a balance between efficient energy consumption and meeting performance metrics [14].

In the majority of crops in Colombia, there are problems in the growth of the plants, the development of their fruit, or in relation to diseases that generate infections.

Additionally, the causes of these problems are unknown due to the scarce or inexistent monitoring of the crops. Moreover, the ideal conditions on which the type of plantation should grow and the incidence of external factors are not considered. Therefore, in this study, we propose a continuous supervision system for an agronomic crop considering the environmental variables and conditions of the site. To determine the efficiency and effectiveness of a WSN in a cacao crop, we performed measurements and transmission of test data in a controlled environment (namely, an open field) to achieve a connection between the modules with line of sight. Additionally, we conducted a factorial experiment to determine the incidence of 3 characteristic parameters (factors) of the WSN on two performance outputs.

This article is organized as follows. The context of the problem and a review of the literature were presented in the Introduction.

Next, the Material and Methods section describe the system architecture and the experimental design. Afterward, the results and collected data are presented and analyzed. Finally, the conclusions of this study are drawn from the results. 


\section{METHODOLOGY}

\subsection{Wireless Sensor Networks}

The wireless networks supported by the ZigBee protocol have three types of devices to regulate their operation:

- Coordinator. Every ZigBee network always has a single coordinator. This node is responsible for creating the network, managing addresses, and managing other functions that define the network, its security, and its adequate performance.

- Router. This node has all the features of the ZigBee protocol. The router can join the existing network to send, receive, and route information to the coordinator node.

It acts as a messenger for the communication between other devices that are too far away to transmit information on their own. A network must have multiple router nodes with a constant power source since they should operate at all times.

End device. It is essentially a reduced version of a router node. It can join the existing network to send and receive messages, but it cannot act as messenger between other devices. As a result, the end device uses less hardware resources and turns itself off automatically for time intervals using a sleep mode.

For the implementation of a wireless network, it is necessary to define the topology that will be used, considering the location of the access points or gateways and the minimum distance from the network infrastructure to the node or final device [15]. In this study, we used the Cluster tree topology because of the surrounding environment. In this topology, the routers form a backbone network with final devices grouped around each router.

Its configuration is very similar to a mesh topology. It is also important to determine the position of the nodes in relation to the obstacles; in this case, the plants and the ground, as well as the distance between the nodes and the coordinator. In [16], the authors tested the incidence of distance and obstacles on the radio link between a coordinator and an end device by examining the number of packets successfully received. Their purpose was to analyze the feasibility of implementing XBee technology in wireless accelerometric sensors (WAS) for monitoring elastic waves on soil surfaces [16].

In order to establish the WSN, we built 7 nodes; 3 of them are end devices, 3 are routers, and one node is the coordinator.

The architecture of the system is shown in Fig. 1.

The Raspberry Pi 3 model $\mathrm{B}$ card in Fig. 1 is the core of the system. It is responsible for receiving the information from the sensor network using an XBee module connected through a serial port.

The Raspberry $\mathrm{Pi}$ and the XBee module work together as the Coordinator node of the network and use the Zigbee protocol to receive the information. Table 1 shows the components of each type of node, and Fig. 2 illustrates the cluster tree topology.

Table 1. Types of nodes

Source: Created by the authors.

\begin{tabular}{lc}
\hline \multicolumn{1}{c}{ Node type } & Components \\
\hline Coordinator & Raspberry Pi + XBee module \\
Router* & Microcontroller + XBee module + \\
& sensors \\
End device* & Microcontroller + XBee module + \\
& sensors \\
\hline
\end{tabular}

"The difference between Router and End device is energy consumption. The End device is in sleep mode most of the time, while the Router must be operating at all times.

Each router and end device consist of an XBee module, an ATMega-328P Microcontroller, and environmental sensors. The data from the sensors are processed by the microcontroller and sent to the Coordinator through the XBee module using the ZigBee protocol. Additionally, the system includes a mobile application developed in Android for the supervision of the environmental variables of each of the 
supervised plants. Fig. 3 outlines the interaction among different software tools that run on different devices in the system.

The Raspberry Pi 3 model B has an interpreter for Python programming language. We developed an algorithm in Python for processing and storing the data coming from the XBee modules. The database, where the data are stored, requires the Structured Query Language, which is interpreted and organized by the database manager MySQL. To develop a service that allows queries through Web ports, the $\mathrm{PHP}$ non-interpreted language was used. This service enabled us to post GET queries to the database and allowed the responses to be interpreted and schematized by the Android Mobile App.

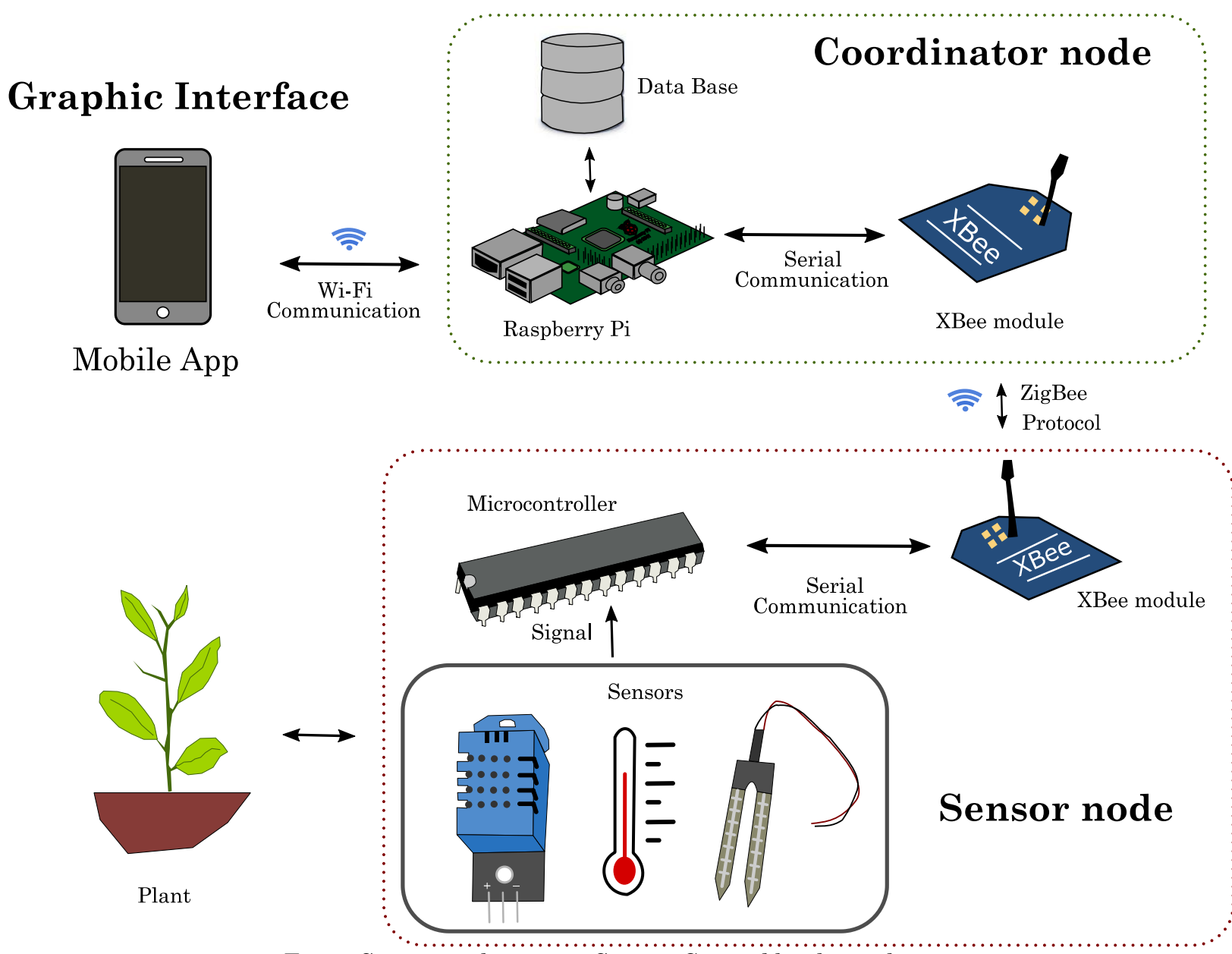

Fig. 1. System architecture. Source: Created by the authors. 

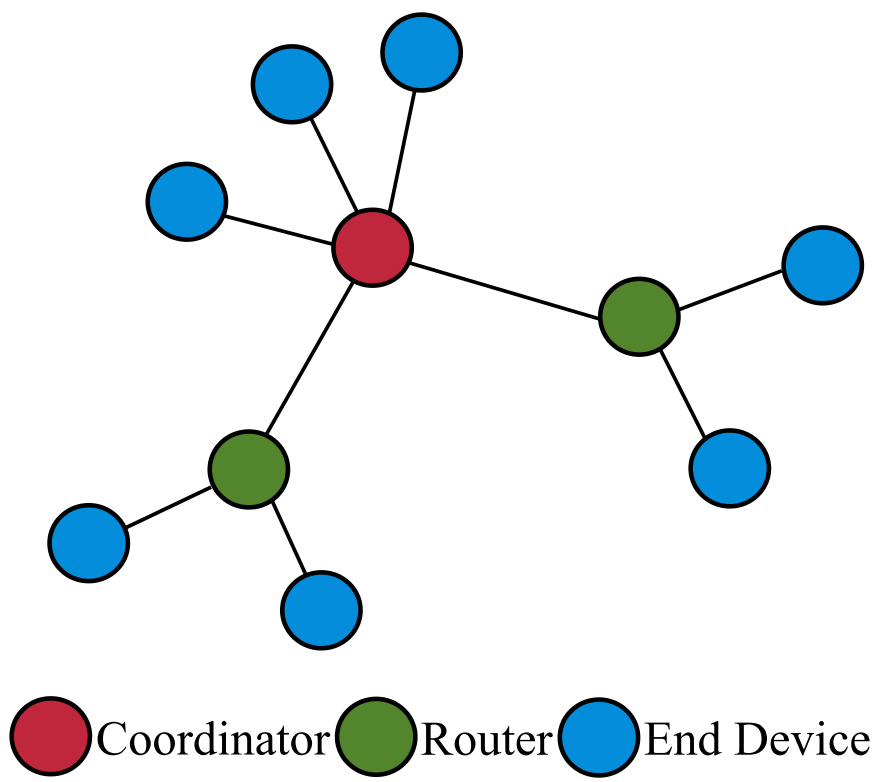

Fig. 2. Cluster tree network topology Source: Created by the authors.
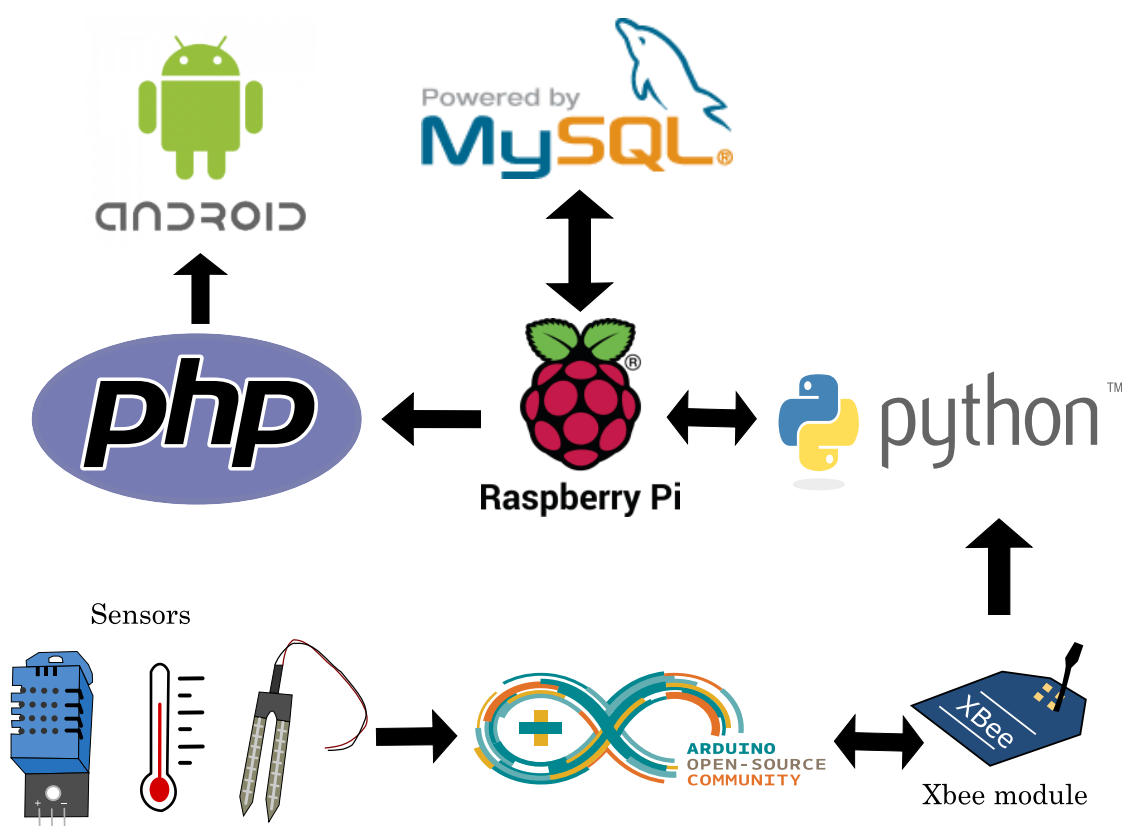

Fig. 3. Software tools to interface the system components Source: Created by the authors.

In each sensor node (router or end device), an ATMega 328P microcontroller was used for conditioning the signal of the sensors and processing the data. We used Arduino IDE to develop the firmware algorithm of the microcontroller, and the
XBee modules were configured in API mode for the transmission and reception of data.

Furthermore, we used open access libraries available online and developed by the Arduino and Raspberry Pi communities.

\subsection{Factorial experiment}


We conducted a factorial experiment to determine the incidence, on two performance outputs, of three characteristic parameters (factors) of the WSN:

-Height with respect to the ground (0.5, $1,1.25$, and 1.8 meters)

-Distance between transmitter and receiver $(5,10,20$, and 40 meters)

-Type of antenna (Wire or PCB)

The two performance outputs of the WSN are Received Signal Strength Indicator (RSSI) and data transfer time (defined here as the time for a transmission to be sent and acknowledged between the transmitter and the final receiver). To measure the data transfer time, we started a timer in the microcontroller of the router, transmitting 71 bytes to the coordinator, and stopped the timer when the microcontroller received the acknowledgment signal from the coordinator.

The experiment consisted of three factors; two of them had four levels, and the other one had two levels (422 factorial design). The purpose of the experiment was to find the best combination of factors to maximize the RSSI and minimize data transfer time.

These parameters (factors) were considered to determine the optimal position of the sensor nodes in the cocoa crop. However, in this section, we refer to an experiment in an open field to establish how close the node sensors should be so that the signal is strong enough and all the data gathered from the sensors can be processed in the network. The type of antenna was also evaluated to determine whether there was an improvement in the RSSI when a certain kind was used.

To ensure accuracy in the measurement, we followed the ITU-R SM.378 recommendation which states:

"For high reliability estimation of the expected field strength at a point at a given distance from a transmitter, one should know the spatial distribution of the field strength in the environment local to the point of measurement. To that end measurements should be taken at several points on a demarcated area. Based on a normal distribution the required number of samples, for a certain degree of reliability, that the field strength lies within a certain range of values around the expected field strength, depends on the standard deviation $\sigma$. By finding the best and worst reception points of that area Emax and Emin can be measured. Based on practical experience an estimate of the standard deviation can be obtained using: Emax $\operatorname{Emin}=5 \sigma "$ [17].

According to the recommendation, the number of measurements that must be taken to increase the level of confidence depends on the minimum and maximum values of the measured intensity level. In our field measurements, the maximum difference never exceeded $10 \mathrm{~dB}$; hence, 15 measurements should be obtained at the same point to guarantee a confidence level of $95 \%$. These values were used to analyze the factorial experiment using Minitab software.

\section{RESULTS AND DISCUSSION}

The XBee modules can be configured in two ways to send data: "Transparent" mode or "API" mode. In the first one, the XBee modules communicate with each other directly in an open channel with no data structure, codification or encryption; therefore, communication is not reliable.

However, building a network to communicate several devices with each other requires the "API" mode, which allows us to send and receive data using "packets" through predefined data structures. Therefore, all the modules used in this study were configured in API mode to build the network and manage them with open access libraries. 


\subsection{Building the sensor nodes}

In this work, six sensor nodes were built. Each one consisted of the following components:

-ATMega 328P microcontroller

-XBee S2C module

-Li-Po 3300-mAh battery

-Customized electronic card for signal conditioning and power regulation between the power supply battery and the aforementioned components, with connection ports for the sensors

-Four sensors to measure temperature, relative humidity, soil moisture, UltraViolet (UV) light, and visible light intensity

-Custom-size acrylic case

Every sensor node was placed in a different plant, located in strategic positions taking into account the parameters of distance, shadows, and access to irrigation. Fig. 4 is a picture of the sensor nodes that were built for this application. Each node is capable of measuring five environmental variables in the crop (temperature, humidity, soil moisture, UV light, and visible light intensity) and transmitting data wirelessly using ZigBee technology. The Li-Po battery provides electric energy to the nodes to guarantee an autonomy of at least 14 days (actual energy consumption varies according to sleep time mode and operating conditions).

\subsection{Location of the nodes in the crop}

When all the individual components of the system were complete, the assembly followed these steps:

-Locate the sensors to measure the variables.

-Configure the sensor network according to the selected topology.

-Link the sensors with the server and the database.
-Connect the system with the user interface for monitoring.

This study was conducted in an agronomic crop of cacao with an extension of 1.77 hectares, a perimeter of 562 meters, and approximately 1000 plants. The plants were separated 3 meters from each other in all directions and irrigated by furrows, with the same distance, that transport water by gravity. Every plant was between 1.8 and 3 meters tall with branches at different heights and an average age of 1 year.

The coordinates of the crop, located in a rural area, are $7^{\circ} 54^{\prime} 29.52^{\prime}$ North Latitude and $-72^{\circ} 37^{\prime} 16.788^{\prime \prime}$ West Longitude. Fig. 5 illustrates the geographical location of the crop, the selected measurement area, the location of the sensor nodes, and the surrounding geography. The total area of the crop is 2.7 hectares; the monitoring system covers about $3 \%$ of the crop area; the image and locations were obtained with Google Maps Web tool.

As mentioned before, the locations of the sensor nodes were determined by the distance to the coordinator, shadowed areas, and access to irrigation. These parameters were considered to have diversity in the measurements of the variables among different plants. Based on this, we established which nodes should be routers so they can provide a path to reach the coordinator if the farthest nodes could not do it directly. Three sensor nodes were defined as Routers and located at an adequate distance to allow communication with the Coordinator and thus cover the three End Device nodes, as well as the target area of the crop. Fig. 5 shows the connection of the End Devices to the Routers, and their link to the coordinator.

In the figure, End Devices are blue; Routers, red; and the Coordinator, green.

The distance between the Coordinator and Router 1 is approximately 23 meters; and between Router 2 and End Device 1, 21 meters. 


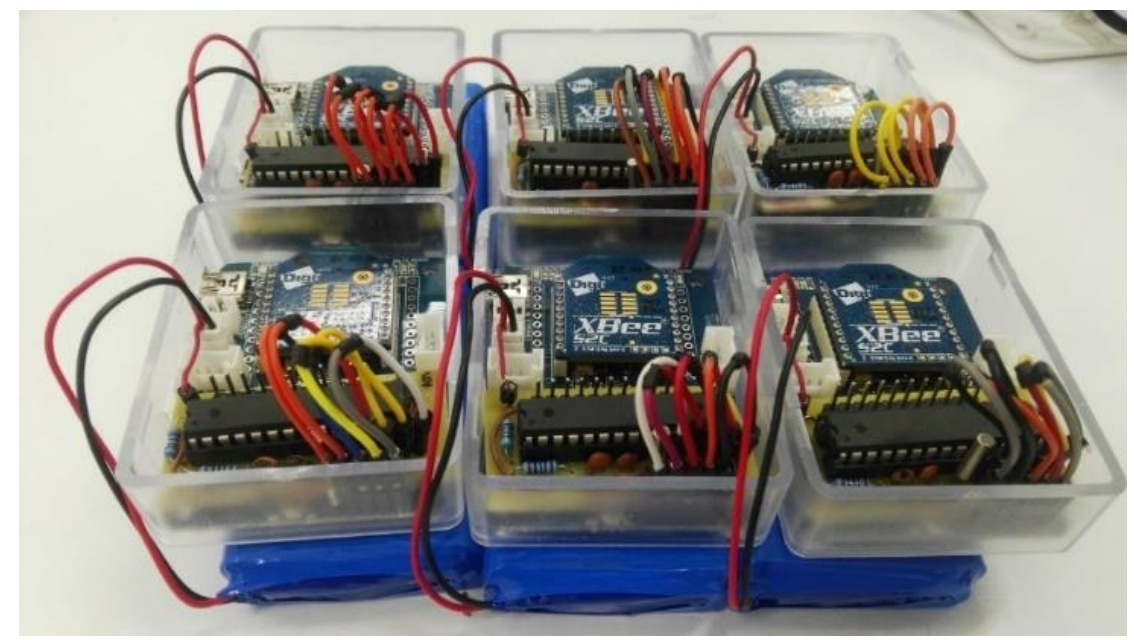

Fig. 4. Zigbee wireless sensor nodes Source: Created by the authors.

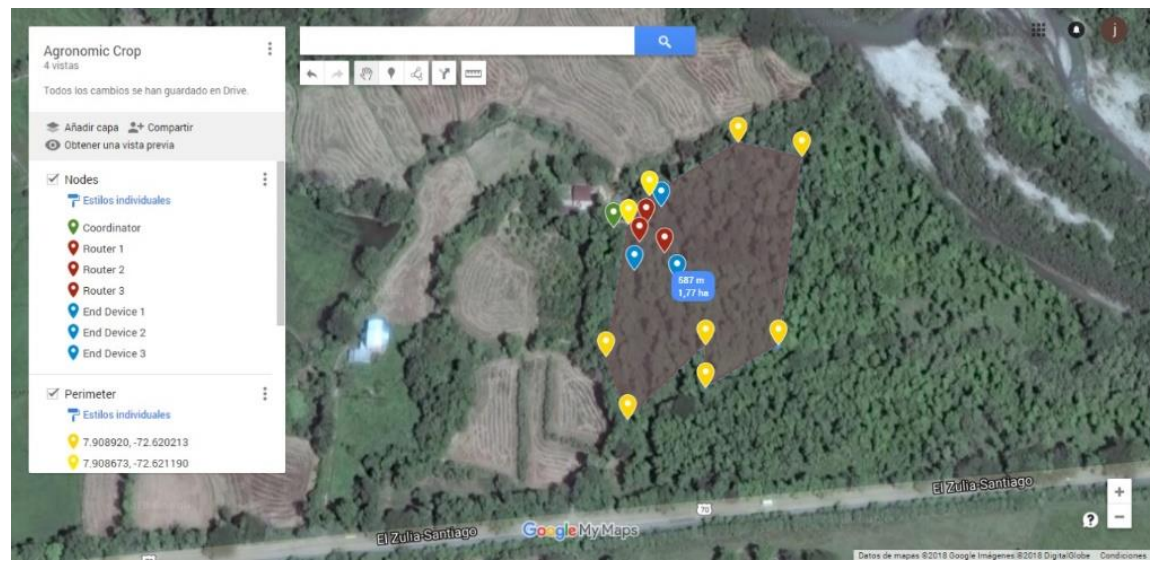

Fig. 5. Map showing the location of sensor nodes in the cocoa crop Source: Google Maps.

\subsection{Coordinator Firmware}

As explained above, the Coordinator node is composed of a Raspberry $\mathrm{Pi}$ and an XBee module. The Raspberry Pi hosts the database where the data from all the sensors are stored. The XBee module receives the packets, de-encapsulates them, and sends them to the Raspberry Pi to be stored in the corresponding tables of the database. We developed a Python script to manage the General Purpose Inputs and Outputs (GPIO) of the Raspberry Pi.

Multiple open access libraries are available in Python for handling XBee modules; for this system, we used the XBee library, copyrighted (c) by Lauri Pesonen, 2014.

\subsection{Line-of-sight measurements}

To determine the efficiency and effectiveness of the WSN, we performed measurements and transmitted test data in a controlled environment (namely, an open field) to establish a connection between the modules with line of sight.

After running the factorial experiment in Minitab, we obtained the combination of parameters that have the highest effect on the outputs (RSSI and data transfer time).

In Fig. 6, the type of antenna is the factor with the highest effect on the RSSI as an individual parameter. That is, the strength of the signal varies substantially with the different types of antenna, and 
this change is perceived more than with the modification of any other individual parameter or combination of parameters.

The next factor with a significant influence is, as expected, the distance between the transmitter and the receiver.

Finally, the least influential parameter on the RSSI output value was the height of the node with respect to ground; the effect of this parameter was weak either individually or in combination with the type of antenna. This does not mean that the height of the antenna with respect to ground does not influence the variation of the intensity of the received power; it means that its effect is less strong than that of the other factors. In our analysis, we also found that, at heights above 1.25 meters, the change in the intensity level is not significant because, when the height of the antenna is exceeded, the ground effect on the signal decreases drastically. These values have a confidence level of $95 \%$ $(\alpha=0.05)$.

Additionally, Fig. 7 can be used to analyze the incidence of each factor on the RSSI. The received signal considerably improves if the wire antenna is used instead of its PCB counterpart. Moreover, as the distance between transmitter and receiver increases, the level of the RSSI value decreases due to the propagation distance, except between 5 and 10 meters.

Finally, we calculated the optimal height of the antenna from the ground to avoid the ground effect, considering the frequency of the wave.

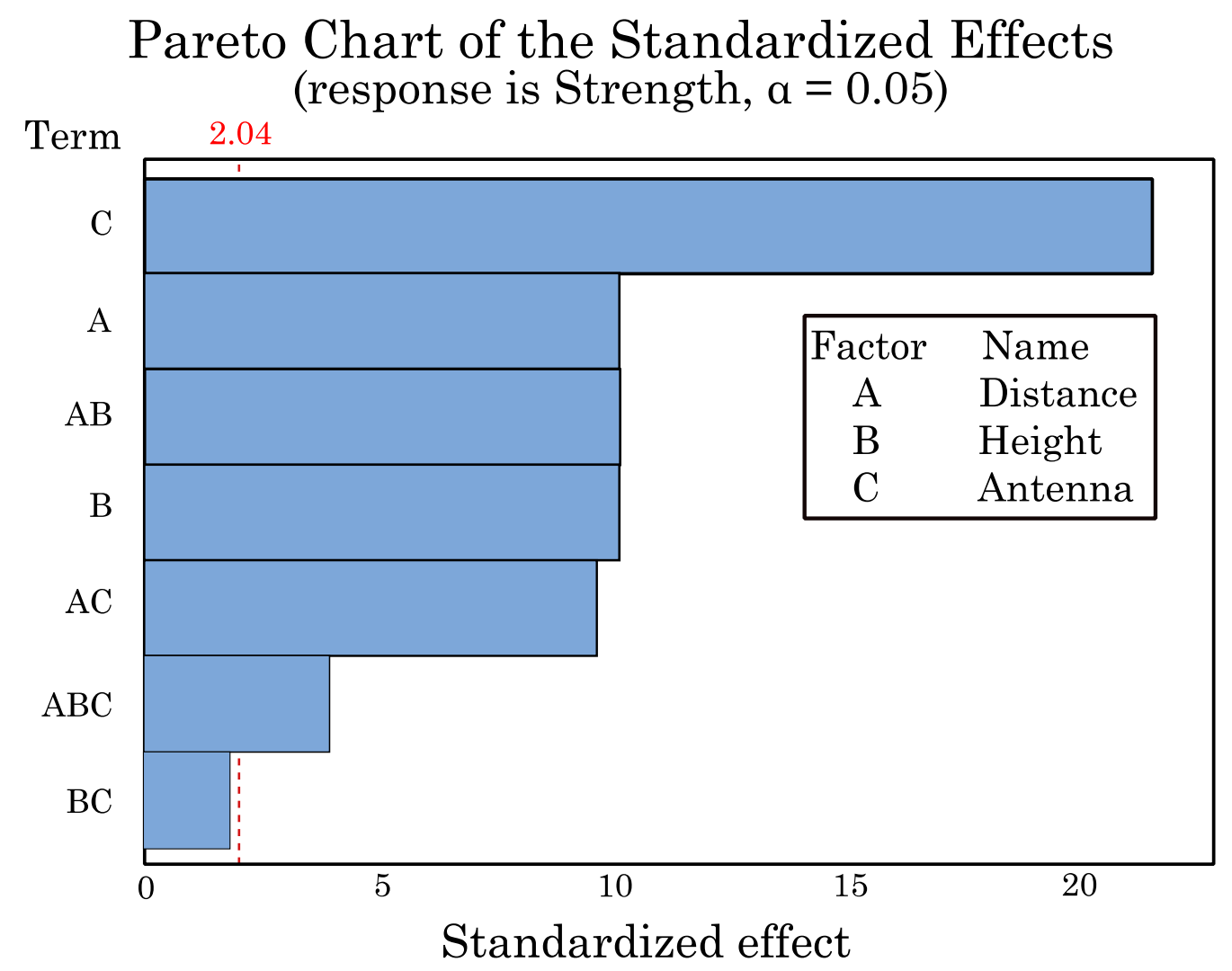

Fig. 6. Incidence of factors on RSSI

Source: Created by the authors. 


\section{Main Effects Plot for RSSI Fitted Main}

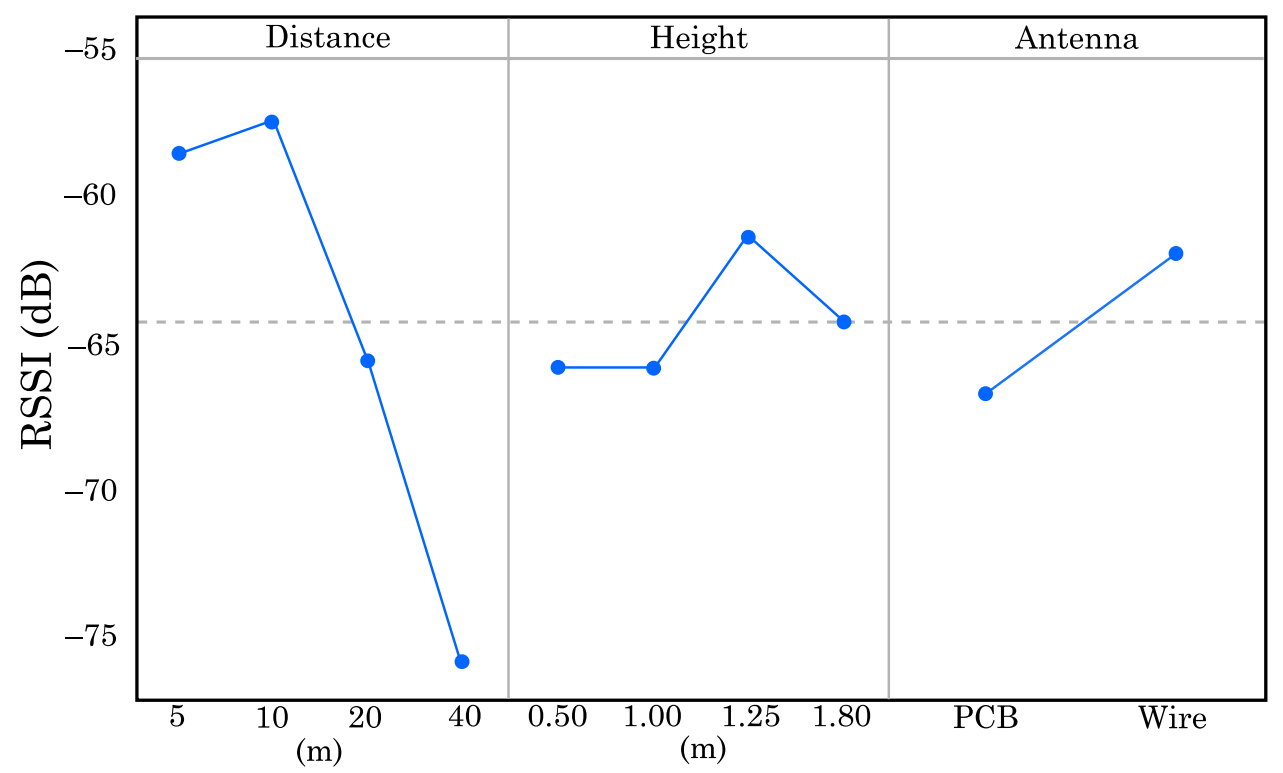

Fig. 7. Effects of the factors on RSSI. Source: Created by the authors.

Fig. 8 presents the analysis of the factorial design for the time output. The strongest effect on the data transfer time is caused by the combination of distance between nodes and height from the ground.

As the distance between the transmitter and receiver grows, the travel time is longer and signal multipath occurs due to the propagation phenomenon. This results in the same radio signals reaching the receiving antenna following two or more paths.

The second most significant effect on data transfer time is produced by the combination of the 3 factors. Finally, the factor with the weakest effect on data transfer time is the type of antenna because the intensity of the signal does not affect the time. As expected, data transfer time is affected mostly by the distance and the multipath delay between the XBee modules.Fig. 9 illustrates the relationship between each factor and data transfer time.

The variation in time (in the range of milliseconds) caused by each type of antenna is not significant; furthermore, there is a non-stable fluctuation of such time with an increase in distance. However, antenna height has a direct incidence on data transfer time, as occurred with RSSI.

Therefore, antenna height is one of the most important factors that should be considered due to the ground effect and the resulting multipath signal components after one or more reflections on the ground, the trunk of a tree, or another object.

\subsection{Measurements in the cocoa crop}

Based on the open field experiment with line of sight (Section 3.4), we determined that the height required to avoid the ground effect was above one meter.

Therefore, a fixed height of 1.25 meters was defined for the experiment in the cocoa plantation.

As opposed to the previous experiment, the stems and branches of the plants in the crop can act as obstacles and signal dispersion may occur. Once again, we performed a statistical analysis of factorial design, but only two factors were considered, namely, distance between nodes and type of antenna. 


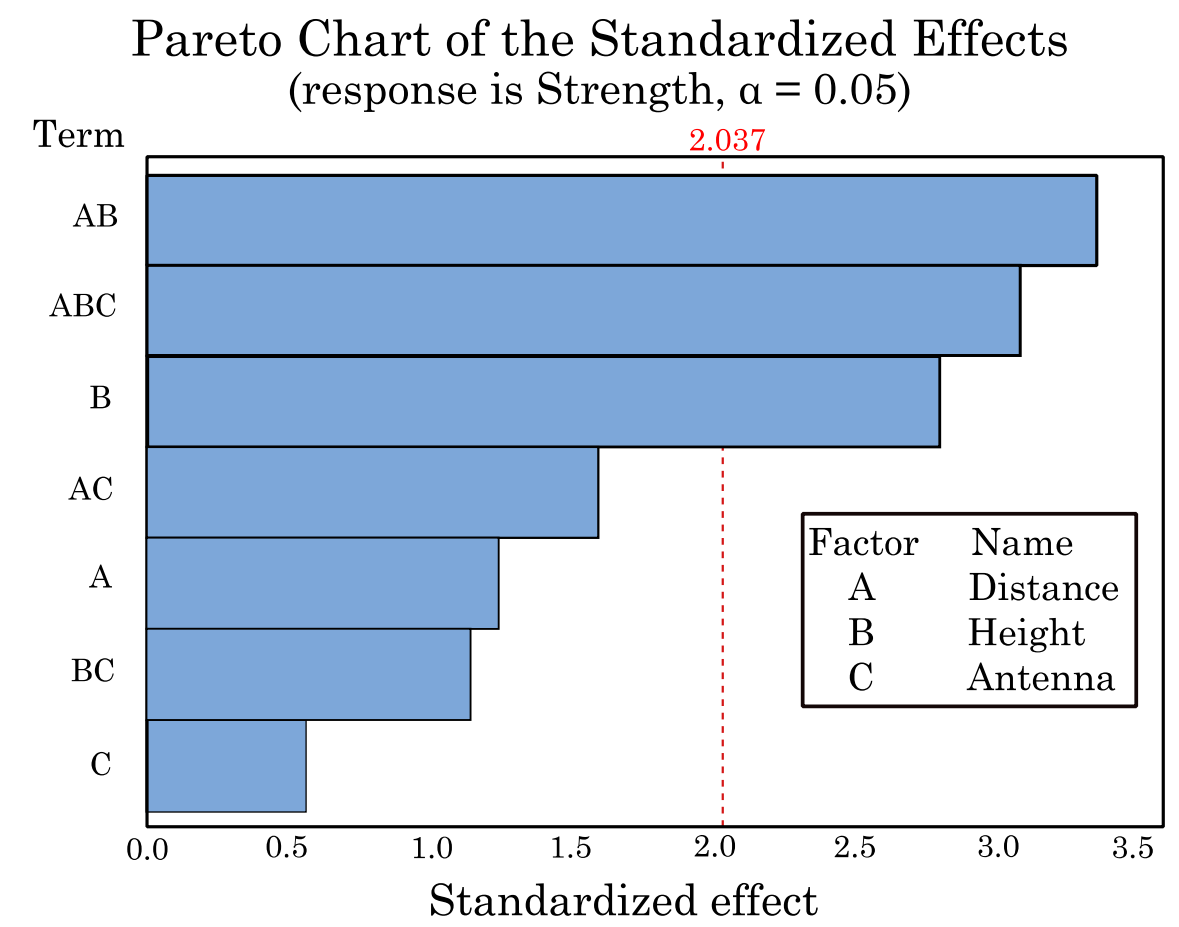

Fig. 8. Incidence of factors on data transfer time Source: Created by the authors.

\section{Main Effects Plot for Data Transfer Time Fitted Main}

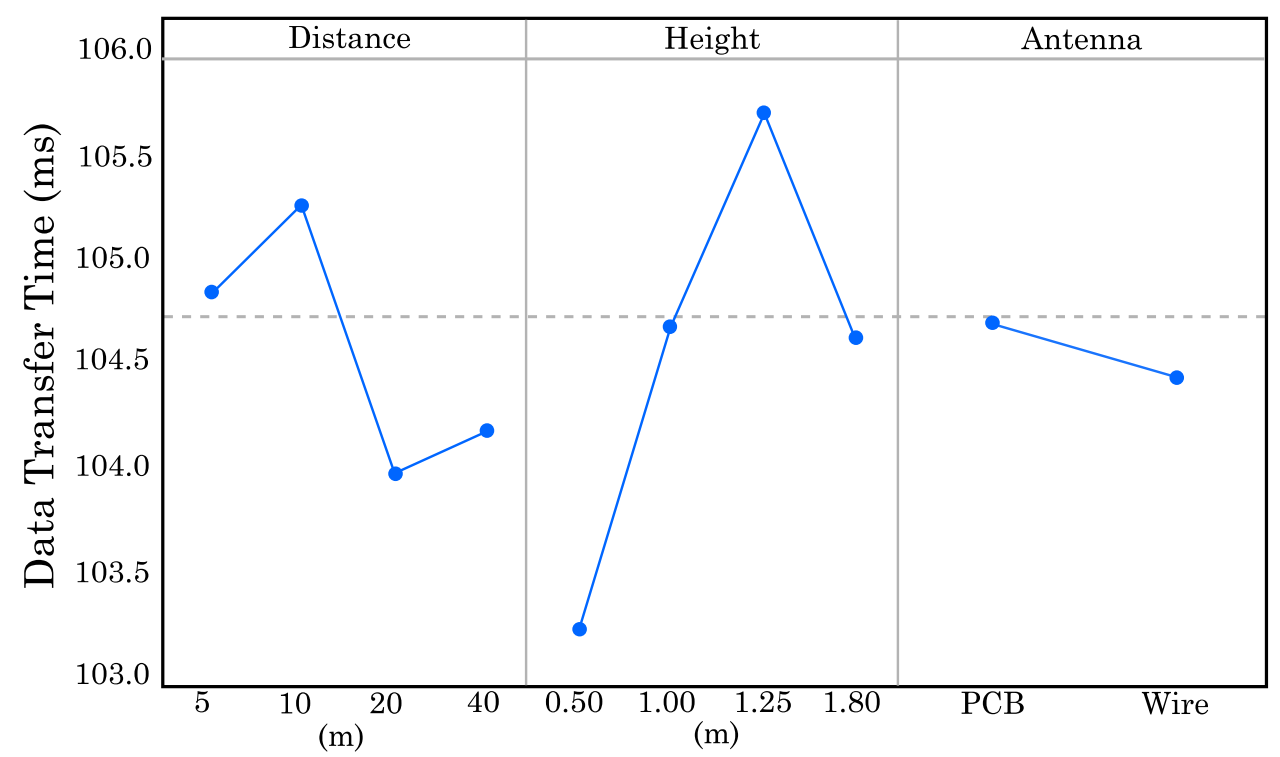

Fig. 9. Effects of the factors on data transfer time

Source: Created by the authors. 
Fig. 10 shows the relationship between distance between nodes and RSSI for both types of antenna. As expected, RSSI decreases as the distance increases, reaching values below $-90 \mathrm{~dB}$, which is a very low signal intensity, close to complete signal loss. The intensity of the signal is always better with the wire antenna, although both options offer the same performance at 20 meters.

A similar analysis was performed for data transfer time. Fig.11 presents the incidence of type of antenna and distance on said time. The plot is relatively flat during the first 35 meters, even with different types of antenna. Nevertheless, after 35 meters, the data transfer time increases more than twice due to the loss of data caused by a decrease in the signal intensity at long distances, as observed from the RSSI results (Fig. 11).

The aforementioned factorial experiments allowed us to establish an ideal distance between the sensor nodes in the WSN to guarantee the communication of the measured variables with short times and negligible data loss: between 20 and 30 meters (either between End Devices and Router, or between Router and Coordinator).

The sensor nodes were located as shown in Fig. 5. Each node measures temperature, humidity, soil moisture, UV light, and visible light intensity. These variables affect the development of the plants' physiology. An in-depth analysis of the growing conditions and physiology of the cocoa plants is currently being conducted by PLANTAE research group from the Department of Agriculture Sciences at Francisco de Paula Santander University.

Their study includes the correlation of the environmental variables and the growth of the plant, the fruits, and the evolution of specific diseases that affect some plants. When this article was submitted, the sensor nodes had been collecting data for about two months.

\section{Interaction Plots for RSSI \\ Fitted Means}

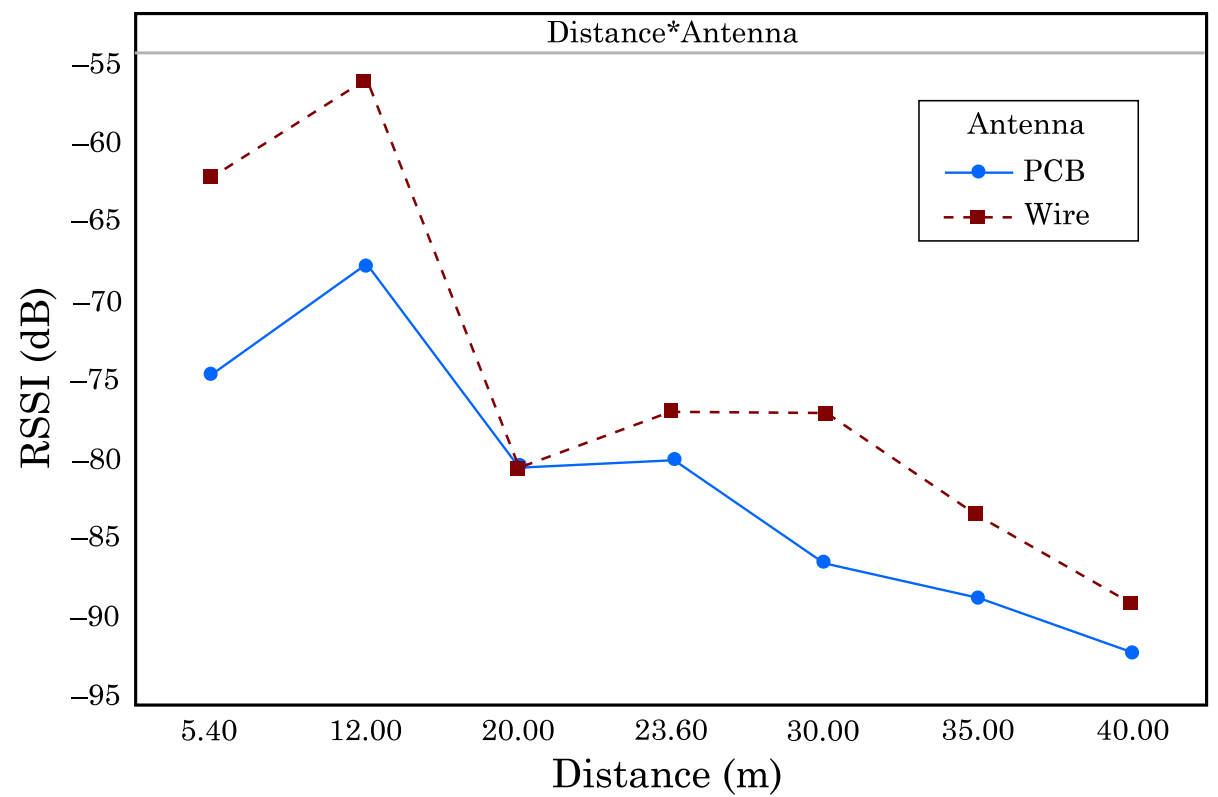

Fig. 10. Relationship between RSSI and the distance between the two modules using two types of antenna. Source: Created by the authors. 


\section{Interaction Plots for Data Transfer Time Fitted Means}

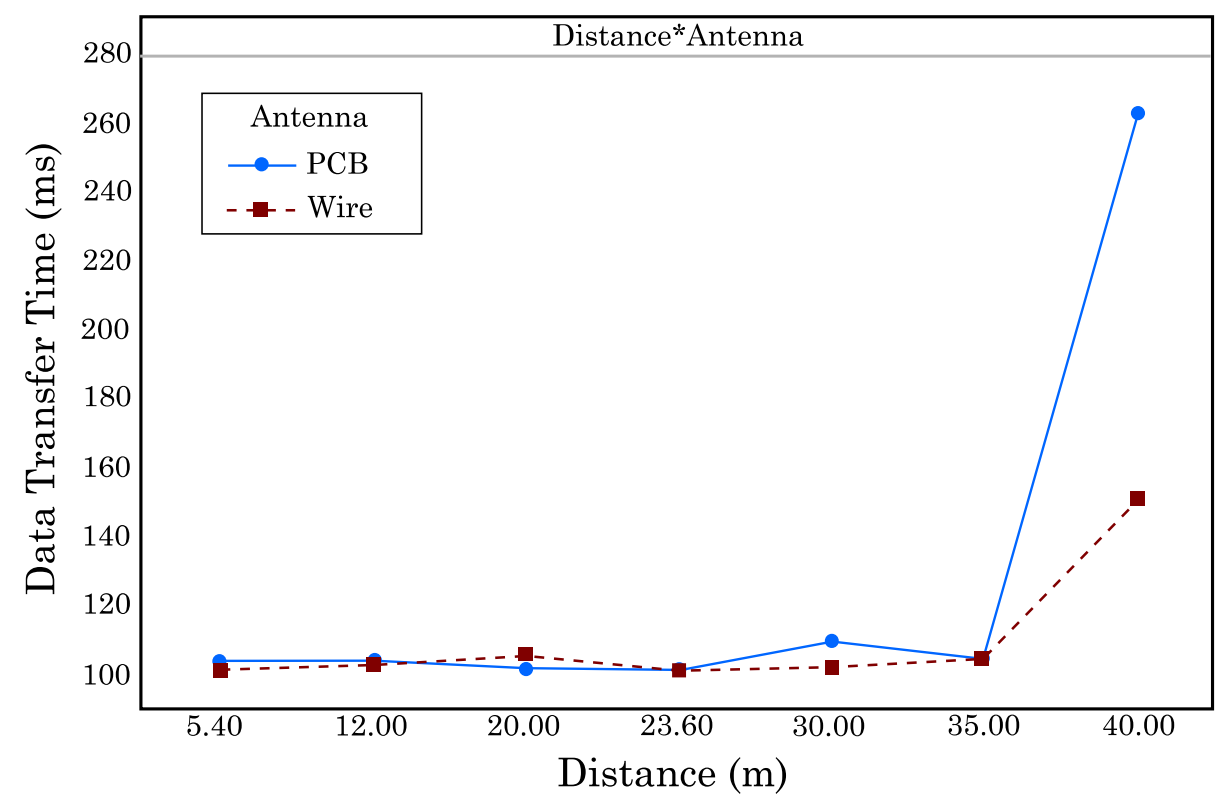

Fig. 11. Analysis of the incidence of different factors on data transfer time Source: Created by the authors.

\section{CONCLUSIONS}

-The design of a Wireless Sensor Network using factorial experiments was presented in this work. We considered three factors: height of sensor nodes from the ground (4 levels), distance between nodes (4 levels), and type of antenna (2 levels). We also analyzed RSSI and data transfer time as performance parameters. The factor with the strongest effect on RSSI is the type of antenna, and the wire antenna achieved an $8 \%$ higher RSSI than its PCB counterpart.

Furthermore, the calculations of antenna height and field measurements were in good agreement.

-The strongest effect on data transfer time is produced by the combination of two factors: distance between sensor nodes (i.e., XBee modules) and height of the sensor node from the ground. The shortest time was achieved with an average height of 0.5 $\mathrm{m}$ and an average distance of $20 \mathrm{~m}$ between nodes. However, the maximum difference in data transfer time in all the experiments was below $3 \mathrm{~ms}$, which is negligible for monitoring environmental variables in a crop.

-After running factorial experiments in two scenarios (namely, open field and cocoa crop) lower RSSI and longer data transfer time values were measured in the cocoa plantation due to signal dispersion caused by the stems, branches and leaves of the plants. Despite a lower RSSI in the agronomic crop environment, we demonstrated that the WSN can fully operate with node sensors separated $20 \mathrm{~m}$ to $30 \mathrm{~m}$ from each other. Additionally, the difference in data transfer time between the crop field and the open field is less than 300 milliseconds, which is not significant for monitoring environmental variables in agronomic applications. With these experiments, we proved that data are not lost in crops, in part due to the multiple attempts of the XBee modules to establish a connection with the nearest module.

-The proposed methodology and WSN monitoring system can be used for agronomic applications in rural areas in Colombia to increase crop yields, thus 
maximizing agricultural productivity. They can help us understand and deal with the outset of plant diseases as well as establish the optimal environmental conditions for the healthy growth of different agricultural products.

\section{REFERENCES}

[1] J. Azcón-Bieto y M. Talón, Fundamentos de Fisiología Vegetal, Universidad de Barcelona: McGraw-Hill, 2008. Available: URL

[2] C. A. Vera Romero, J. E. Barbosa Jaimes, and D. C. Pabón González, "Acople de sensores en la medición de variables ambientales usando tecnología ZigBee," Sci. Tech., vol. 19, no. 4, pp. 419-424, Dec. 2014. Available: URL

[3] Y. Li, X. Guo, R.-H. Shi, and F.-L. Yang, "Monitor and Control Wireless Sensor Nodes by B/S Architecture," in 2014 International Conference on Wireless Communication and Sensor Network, Wuhan, 2014. pp. 204-206 https://doi.org/10.1109/WCSN.2014.48

[4] J. F. Monsalve-Posada, A. Arias-Londoño, and J. G. Mejía-Arango, "Desempeño de redes inalámbricas y redes industriales inalámbricas en procesos de control en tiempo real bajo ambientes industriales," TecnoLógicas, vol. 18, no. 34, p. 87, Jan. 2015. https://doi.org/10.22430/22565337.215

[5] M. Lee, J. Hwang, and H. Yoe, "Agricultural Production System Based on IoT," in 2013 IEEE 16th International Conference on Computational Science and Engineering, Sydney, 2013. pp. 833-837. https://doi.org/10.1109/CSE.2013.126

[6] J. Ma, X. Zhou, S. Li, and Z. Li, "Connecting Agriculture to the Internet of Things through Sensor Networks," in 2011 International Conference on Internet of Things and 4th International Conference on Cyber, Physical and Social Computing, Dalian, 2011. pp. $184-187$. https://doi.org/10.1109/iThings/CPSCom.2011. 32

[7] Liang-Ying, G. Yun-feng, and Zhao-Wei, "Greenhouse environment monitoring system design based on WSN and GPRS networks," in 2015 IEEE International Conference on Cyber Technology in Automation, Control, and Intelligent Systems (CYBER), Shenyang, 2015. pp. 795-798.

https://doi.org/10.1109/CYBER.2015.7288044
[8] A. Cama-Pinto, F. Gil-Montoya, J. GómezLópez, A. García-Cruz, and F. ManzanoAgugliaro, "Wireless surveillance sytem for greenhouse crops," Dyna, vol. 81, no. 184, pp. 164-170, Apr. 2014.

https://doi.org/10.15446/dyna.v81n184.37034

[9] S. Ferdoush and X. Li, "Wireless Sensor Network System Design Using Raspberry Pi and Arduino for Environmental Monitoring Applications," Procedia Comput. Sci., vol. 34, pp. 103-110, 2014. https://doi.org/10.1016/j.procs.2014.07.059

[10] M. S. Azimi Mahmud, S. Buyamin, M. M. Mokji, and M. S. Z. Abidin, "Internet of Things based Smart Environmental Monitoring for Mushroom Cultivation," Indones. J. Electr. Eng. Comput. Sci., vol. 10, no. 3, pp. 847-852, Jun. 2018. Available: URL

[11] M. S. M, S. Das, S. Heble, U. Raj, and R. Karthik, "Internet of Things based Wireless Plant Sensor for Smart Farming," Indones. J. Electr. Eng. Comput. Sci., vol. 10, no. 2, pp. 456-468, May 2018. Available: URL

[12] W. T. Sung, J. H. Chen, C. L. Hsiao, and J. S. Lin, "Multi-sensors Data Fusion Based on Arduino Board and XBee Module Technology," in 2014 International Symposium on Computer, Consumer and Control, Taichung, 2014. pp. 422-425. https://doi.org/10.1109/IS3C.2014.117

[13] M. Pule, A. Yahya, and J. Chuma, "Wireless sensor networks: A survey on monitoring water quality," J. Appl. Res. Technol., vol. 15, no. 6, pp. 562-570, Dec. 2017. https://doi.org/10.1016/j.jart.2017.07.00

[14] J. C. Correa-Chica, J. F. Botero-Vega, and N. Gaviria-Gómez, "Energy consumption and quality of service in WBAN: A performance evaluation between cross-layer and IEEE802.15.4," DYNA, vol. 84, no. 202, pp. 120-128, Jul. 2017. https://doi.org/10.15446/dyna.v84n202.61895

[15] C. M. Durán-Acevedo and H. L. García-Sierra, "Desarrollo de un Sistema Inalámbrico para la Supervisión y Control de un Aerogenerador," TecnoLógicas, pp. 395-409, Oct. 2013.

https://doi.org/10.22430/22565337.331

[16] M. R. Fulla, J. L. Palacio-Bedoya, C. A. Flórez-Velásquez, and V. H. AristizábalTique, "Módulo Inalámbrico para el Sensado de Vibraciones Superficiales en Suelos," TecnoLógicas, vol. Special Ed, pp. 451-464, Oct. 2013.

https://doi.org/10.22430/22565337.352

[17] International Telecommunication Union, "Spectrum Monitoring (Handbook)." 2011. Available: $\underline{\mathrm{URL}}$ 